IOSR Journal of Pharmacy

e-ISSN: 2250-3013, p-ISSN: 2319-4219, www.iosrphr.org

Vol. 2, Issue 6, Nov-Dec. 2012, PP. 20-22

\title{
Compound Palmar Ganglion: A Case Report
}

\author{
Daya Krishna $^{1}$, R.S Bisht ${ }^{2}$, Vikas Sikarwar ${ }^{2}$, Shubash Chand ${ }^{1}$ \\ ${ }^{I}$ Department of Orthopedics; Veer Chandra Singh Garhwali Govt. Medical Science and Research Institute, \\ Srinagar, UK, India \\ ${ }^{2}$ Department of E.N.T and Head \& Neck Surgery; Veer Chandra Singh Garhwali Govt. Medical Science and \\ Research Institute, Srinagar, UK, India.
}

\begin{abstract}
Compound palmar ganglion or tuberculous tenosynovitis of flexor tendons of wrist and hand is a rare disease. The incidence of extra-pulmonary tuberculosis including musculoskeletal system has also increased with re-emergence of tuberculosis due to increase in HIV / AIDS cases. We are presenting a case of 16 year old boy who present with a dumbbell shaped fluctuating swelling between flexor surface of right wrist and palm without any previous or present history of pulmonary tuberculosis /contact and trauma. Radiographic and laboratory finding were normal except raised ESR and strongly positive montouxe test. Patient was managed by open biopsy with excision of thickened synovium and removal of multiple rice bodies or melon seeds and sample send for histopathological examination. Histopathological report showed caseous necrosis and epithelial granuloma along with Langhans type giant cell, staining for AFB was negative. ATT was given to patient for 9 months with regular follow-up. Patient gained full range of motion in wrist in five month and there is no underlying disorder in last follow-up at one and half year. The tuberculous tenosynovitis is rare but must be kept in mind as differential diagnosis of chronic tenosynovitis.
\end{abstract}

Keywords:- Tubercular tenosynovitis, compound palmar ganglion, rice bodies, excision biopsy, flexor tendon.

\section{INTRODUCTION}

Tuberculous tenosynovitis of flexor tendons at wrist and palm or compound palmar ganglion is tubercular infection of the tenosynovium of long flexor tendon more common in male and mostly involve dominant limb. ${ }^{1}$ Tuberculous tenosynovitis is rare but it is still a leading cause of chronic tendon sheath infection. The route of infection is either direct inoculation or hematogenous dissemination from a primary focus; lungs, lymph nodes, kidney, ${ }^{1}$ spine and precipitated by immunosuppression, local / systemic steroid, alcoholism, malnutrition and trauma. ${ }^{2}$ The diagnosis of tubercular tenosynovitis is delayed due to multiple identical condition and differential diagnosis with poor clinical awareness which leads to complications.

\section{CASE REPORT}

A 16 years old boy came to our OPD with chief complains of pain and swelling over volar aspect of right wrist since 8 months, discomfort during wrist movement and sometime tingling over radial three and half fingers. The swelling was gradual in onset and progressively enlarged during last 8 months and during this patient was treated by another clinic by aspiration of fluid, FNAC, oral steroid and NSAID but patient did not get relive. The FNAC report shows chronic inflammation. There is no previous history of any trauma, pulmonary tuberculosis and contact, constitutional symptoms of tuberculosis, rheumatoid arthritis and pain in other joint and lymphadenopathy. On local examination there was swelling $6 \mathrm{~cm} \times 2 \mathrm{~cm}$ in size over volar aspect of right wrist which is mildly tender doughy in consistency, compressible extending longitudinally both sides proximal and distal to the flexor retinaculum of the wrist. Cross fluctuation test is positive and there is no distal motor and sensory deficit. Lab investigation showed raised ESR $[40 \mathrm{~mm} / \mathrm{hr}]$ and CRP, Montouxe test / tubercular reaction is strongly positive. There is no significant finding in radiographs of right wrist and chest.

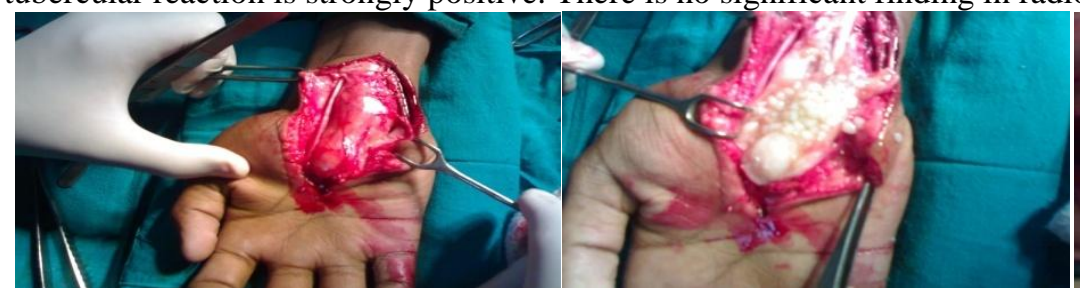

(a)

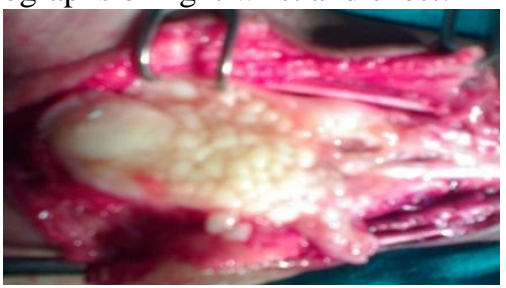

(c) 
Compound Palmar Ganglion: A Case Report

Patient undergoes surgical management [open biopsy] under regional anesthesia and tourniquet at surgery there were multiple rice bodies filled in yellow fluid and thickening of flexor tendon sheath present. Thorough cleaning and tenosynovectomy was done and specimen sent for histopathology and culture sensitivity, a below elbow POP slab applied after closer for 2 weeks. Histopathology report shows caseous necrosis epithelial granuloma with Langhans type of giant cell, Gram and ZN stains of synovial tissue were negative, tissue culture for AFB was positive. ATT was started $2^{\text {nd }}$ day after surgery on the basis of clinical features and pathological appearance and excluding other common conditions. Patient became symptomless after five month with full range of painless movement. We give ATT for a period of 9 month in 2 years follow-up there is no further complains and wrist is fully functional.

\section{DISCUSSION}

The tubercular infection pulmonary/extra-pulmonary is reemerging due to increase in immunity compromising diseases [HIV / AIDS]. The commonly affected extra-pulmonary sites are lymph nodes genitourinary tract, bone marrow, CNS and musculoskeletal system which include bones, joint, bursas and tendons or tenosynovium which is $1.3 \%$ of all patients having tuberculosis. ${ }^{1}$

Tubercular tenosynovitis is more common in upper limb as compare to lower limb and commonly involves the flexor or volar site of wrist and hand of dominant limb. ${ }^{3}$ The pathogenesis may be direct inoculation or heamatogenous spread from a primary focus in lung, lymph nodes, genitourinary tract and bones. ${ }^{1}$ The onset of tubercular tenosynovitis is characteristically insidious and gradual and the progression of the disease is slow. The symptoms are minimum and very mild and the disease is advance till patient seeks treatment. The symptoms of median nerve compression at carpal tunnel may be there in case of huge and tense swelling. ${ }^{4,6,7}$ Lab investigations are mostly negative except raised ESR and strongly positive Montouxe test, radiograph of wrist and chest are mostly normal, MRI shows marked thickening increased vascularity and fluid in synovial sheath of tendons, histopathological examination shows caseous necrosis, epithelial granuloma and Langhan's type giant cells.

There are three histopathological stages of disease depending upon duration of disease, resistance of patient and virulence of infecting agent in early stage there is vascular granulation tissue formation followed by obliteration of tendon sheath by fibrous tissue fluid accumulation and formation of rice bodies or melon seeds which are fibrinous masses or tubercle made by caseation in last stage there is rupture of tendon sheath, extensive caseation and granulation take place. ${ }^{2}$ The rice bodies are also commonly found in many diseases like SLE, rheumatoid arthritis, seronegative arthritis and osteoarthritis of joint. ${ }^{5}$

The differential diagnosis of compound palmar ganglion includes rheumatoid arthritis, gouty arthritis, pyogenic infection, ganglion [infected], sarcoidosis, foreign body tenosynovitis, and fungal infection, pigmented villonodular synovitis of tendon sheath, amyloidosis and synovial chondromatosis. ${ }^{1,2,3,5}$ Such a long list of differential diagnosis and nonspecific clinical, radiological and histopathological finding leads to delay in diagnosis and treatment and resulting into complication. The best way to make a final diagnosis is open biopsy and mycobacterial culture of the pathological material but the culture requires few weeks to diagnose which leads to delay in diagnosis and treatment. According to literature the positive result of synovial fluid AFB staining, culture of mycobacterium, histology and PCR are 32\%, 80\%, 65\% and 63\% respectively and PCR can be used for early diagnosis. ${ }^{1}$ Thus to make a provisional diagnosis of tuberculosis and to start ATT it is always better to rule out other common causes and conclude clinical, radiological, histopathological and PCR findings suggestive of tubercular tenosynovitis.

Combined surgical and medical therapies which include excision of infected tissue, tenosynovectomy followed by ATT for 6 to 9 months give good result and prevent recurrence of disease. A good clinical awareness with keeping tuberculosis as a common cause of chronic tenosynovitis in mind in developing country like India prevent delay in diagnosis and treatment and resulting complication.

\section{REFERENCES}

1). Seiichi Higuchi, Shinichi Ishihara, Hiroyuki Kobayashi and Taidoh Arai: A mass lesion of the wrist: A rare manifestation of tuberculosis. [DOI: 10.2169/internalmedicine.47.0495]

2). Hitesh Lall, Suman K Nag, Vijay K Jain, Rahul Khare and Deepak Mittal: Tuberculous extensor tenosynovitis of the wrist with extensor pollicis longus ruptures: a case report. Journal of medical case reports 2009, 3:142.

3). Pei-Hung Shen, Cheng-Mien Chu, Gua-Shu Huang, Shing-Sheng Wu and Chain-Her Lee tuberculous tenosynovitis of the flexor tendons of the wrist and hand: J Med Sci 2002;22[5]:227-230.

4). Mamoon Rashid, Saad Ur Rehman Sarwar, Ehtesham Ehtesham, Muhammad Zia Ul Islam, Kokab Shah: tubercular tenosynovitis: a cause of carpal tunnel syndrome.JPMA 56:116; 2006.

5). M Tyllianakis, G Kasimatis, S Athanaselis, M Melachrinou: Rice body formation and tenosynovitis of the wrist: a case report. Journal of orthopaedic surgery 2006; 14 [2]:208-11. 
6). A.Gillies tuberculous tenosynovitis of the palmer synovial bursa: compound palmar ganglion. JBJS 193. www.jbjs.org/cgi/reprint13/1/156.

7). Arvind G Kulkarni, Vidyanand V Prabhu. Tuberculosis presenting as carpal tunnel syndrome: a case report with review of literature. http//www.bhj.org/journal/2002-4401-jan/case-102.htm.

8). Tuli SM. Tuberculosis of skeletal system. Jaypee 2004

9). Hsu CY, Lu HC, Shih TT: Tuberculous infection of the wrist: MRI features. AJR Am J Roentgenol 2004,183 [3]:623-628.

10). Regnard PJ, Barry P, Isselin J: Mycobacterium tenosynovitis in the flexor tendons of the hands: A report of five cases. J Hand Surg[Br] 1996,21[3]:351-354. 